\title{
Predicting high utilization of emergency department services among patients with a diagnosis of psychosis in a Medicaid managed care organization
}

Tammy K. Girts

Boehringer Ingelheim Pharmaceuticals, Inc.

Albert G. Crawford

Thomas Jefferson University

Neil I. Goldfarb

Thomas Jefferson University

Mark Bachleda

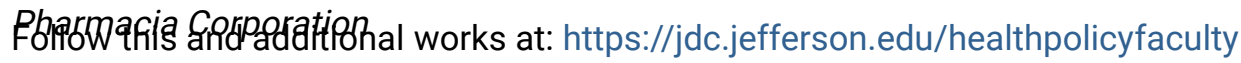

iparogge Health Services Research Commons

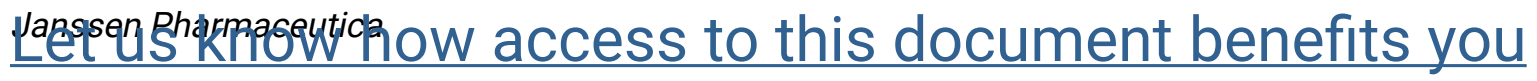

\section{Recommended Citation}

Girts, Tammy K.; Crawford, Albert G.; Goldfarb, Neil I.; Bachleda, Mark; and Grogg, Amy, "Predicting high utilization of emergency department services among patients with a diagnosis of psychosis in a Medicaid managed care organization" (2002). College of Population Health Faculty Papers. Paper 12.

https://jdc.jefferson.edu/healthpolicyfaculty/12

This Article is brought to you for free and open access by the Jefferson Digital Commons. The Jefferson Digital Commons is a service of Thomas Jefferson University's Center for Teaching and Learning (CTL). The Commons is a showcase for Jefferson books and journals, peer-reviewed scholarly publications, unique historical collections from the University archives, and teaching tools. The Jefferson Digital Commons allows researchers and interested readers anywhere in the world to learn about and keep up to date with Jefferson scholarship. This article has been accepted for inclusion in College of Population Health Faculty Papers by an authorized administrator of the Jefferson Digital Commons. For more information, please contact: JeffersonDigitalCommons@jefferson.edu. 


\title{
Predicting High Utilization of Emergency Department Services Among Patients with a Diagnosis of Psychosis in a Medicaid Managed Care Organization
}

\author{
TAMMY K. GIRTS, Pharm.D., ${ }^{1}$ ALBERT G. CRAWFORD, Ph.D., ${ }^{2}$ NEIL I. GOLDFARB, B.A., ${ }^{2}$ \\ MARK BACHLEDA, Pharm.D., ${ }^{3}$ and AMY GROGG, Pharm.D. ${ }^{4}$
}

\begin{abstract}
Studies have demonstrated increased utilization of medical services for patients with behavioral health diagnoses. Medicaid managed care organizations (MMCOs) that operate under behavioral health carve-outs face the challenge of effectively targeting disease management initiatives in the absence of information on behavioral diagnoses. This study sought to develop a predictive model of emergency department (ED) utilization for patients where a diagnosis of psychosis could be identified from a claim associated with a medical service provider visit. A retrospective cohort analysis was performed using medical and pharmacy claims from an MMCO in Philadelphia, Pennsylvania, to identify patients known to have a diagnosis of psychosis and to develop the predictive model. Demographics, comorbidities, medical utilization, and medications were assessed as predictor variables. Within the MMCO, 764 members were identified with at least one medical claim having a psychosis diagnosis. Ordinary least squares multiple regression analysis was performed to measure the correlation between independent variables and ED visits. Variables with significant $F$ ratios in the regression analysis were retained as factors in a risk model to evaluate their additive and cumulative effects. Four variables were significant predictors of high ED utilization: prior number of ED visits, prior number of hospitalizations, history of alcohol abuse, and history of depression. ED utilization increased as the number of risk factors increased: With no risk factors, mean ED use was 0.58 visits (per 6 months), while the cumulative effects of all four factors equated to 8.5 ED visits. The model may be useful to other MMCOs, or similar organizations, seeking to risk-stratify their ED-related disease management activities for patients identified with psychosis.
\end{abstract}

\section{INTRODUCTION}

A LTHOUGH HEALTH CARE spending nationwide has continued to increase annually, the cost of caring for Medicaid enrollees has in- creased at an even higher rate over the last decade. ${ }^{1}$ Total Medicaid program spending approximated $\$ 207$ billion in the fiscal year $2000 .^{2}$ Since 1989, Medicaid expenditures have nearly tripled. ${ }^{1}$ One method state Medicaid programs

\footnotetext{
${ }^{1}$ Health Economics, Boehringer Ingelheim Pharmaceuticals, Inc., Ridgefield, Connecticut.

${ }^{2}$ Office of Health Policy and Clinical Outcomes, Thomas Jefferson University, Philadelphia, Pennsylvania.

${ }^{3}$ Global Pricing \& Market Access Strategy, Pharmacia Corporation, Peapack, New Jersey.

${ }^{4}$ CNS Outcomes Research, Janssen Pharmaceutica, Titusville, New Jersey.
} 
have employed to contain and control costs is to contract with managed care organizations (MCOs) for delivery of health care to their beneficiaries. ${ }^{2,3}$ In 2000, 56\% (18.8 million people) of Medicaid recipients were enrolled in MCOs. ${ }^{3}$ The move from Medicaid fee-for-service to Medicaid managed care has slowed the growth of spending over recent years. There still remain ample opportunities for improving quality and, hopefully by extension, a reduction in expenditures.

Emergency department (ED) services are generally accessible to many patients and can sometimes be overutilized for non-emergency situations, which can contribute to high costs. The Medicaid population is historically prone to problems of access compared with the general population. Many of these patients use the ED as an entry point into the health care system when other venues are more appropriate. ${ }^{4}$ Previous research has shown non-urgent ED use to have an association with factors that are also characteristic of many patients on Medicaid. Specific factors include poor health status, public insurance coverage, fewer weeks of employment, and less education. 5,6

Within an $\mathrm{MCO}$, the primary care physician (PCP) is ultimately responsible for managing the health care of the patient. This model attempts to promote quality and continuity of health care while reducing the amount of duplicative services. Attempts have been made by Medicaid MCOs (MMCOs) to discourage patients from receiving non-urgent care in the ED, but these attempts have shown mixed results. ${ }^{5}$ By identifying and meeting the needs of this population prior to visiting the ED, improved quality of care should follow.

Patients with behavioral health disorders are disproportionately represented in the Medicaid population in terms of prevalence and medical costs. ${ }^{7}$ Typically, behavioral health patients are also high users of emergency services. ${ }^{8-10}$ Targeting intervention services through case management to such a group might be beneficial if their problems can be effectively addressed. 8,10 One of the major goals in community-based case management programs is to promote health and improve the quality of life for the patients. ${ }^{11,12}$ Frequent ED visits and hospitalizations may be viewed as a proxy for the inability to keep patients healthy. It is a widely held belief that these costly services can be reduced to only necessary visits with proper management. ${ }^{11,12}$

\section{Objective}

The primary objective of this study was to develop a predictive model to identify high users of ED services among patients with psychosis in an MMCO. Medical and pharmacy claims were used from an MMCO operating in a mandatory managed care environment.

The following questions were addressed during the process of identifying potential variables to test for in the model: (1) What medical comorbidities are common in patients with psychosis that may affect ED visits? (2) Which types of pharmacological treatment are commonly used to treat patients with psychosis that may affect ED use? (3) What other types of medical resource use may be predictive of ED utilization?

\section{SUBJECTS AND METHODS}

\section{Study design}

A retrospective cohort analysis was performed in patients with psychosis enrolled in a state MMCO in Philadelphia, PA. The data source for the analysis consisted of medical and pharmacy claims from the 1998 calendar year. Claims for medical and pharmacy resource use during the first 6 months of the year, January 1-June 30, were used to predict whether patients would be high users of ED services during the second 6 months of the year, July 1-December 31.

A list of comorbidities that may be contributors to increased use of the ED was developed a priori from published literature and author discussion. A frequency check was run on all medical claim diagnoses to determine if enough data were available to test the chosen comorbidities as variables for ED association. Diseases that were not previously identified but showed a high prevalence in the study population were also used as comorbidity variables and tested for correlation with ED use. The comorbidities analyzed were for chronic condi- 
tions; therefore, to provide more data for model development, claims were used from the entire study year when identifying patient comorbidities.

Medical claims for services provided by the behavioral health organization were not available since the MMCO had a mandate to carveout behavioral health services. However, all prescription data were processed through the MMCO. Approximately 72,000 claims were analyzed in this study: 27,000 medical claims and 45,000 pharmacy claims.

\section{Study population}

Inclusion criteria for the study required patients to have at least one medical claim with a psychotic diagnosis code between 295.xx and 299.xx based on the International Classification of Diseases, 9th Revision, Clinical Modification (ICD-9-CM) ${ }^{13}$ There were 764 patients eligible for this study with a requisite ICD-9-CM code in a primary through quaternary diagnosis field for any type of medical claim. Patients were excluded from this study if they had not been continuously enrolled in the MCO during the study year using the Health Plan Employer Data and Information Set (HEDIS ${ }^{\circledR}$; National Committee on Quality Assurance) definition of continuous enrollment (no more than one gap in enrollment of up to 45 days during the study period). Patients younger than 18 years of age at study initiation also were excluded.

\section{Data extraction}

Demographic variables. Demographic variables included age, gender, and ethnicity.

Medical claim variables. Using claims in the first 6 months of the study (January-June 1998), medical resource use variables included total ED visits as both a continuous variable and dichotomized ( $0-1$ vs. 2 or more visits) to differentiate repeat users. Other medical resource variables were presence of hospitalization, total number of hospitalizations, total hospital days, presence of PCP visit, and the total number of PCP visits. Using claims from the entire study year, comorbidity indicators included diabetes, sickle cell anemia, neurotic disorders, alcohol abuse, other drug use (besides alcohol), depression, essential hypertension (HTN), heart failure, asthma, other lung disease (besides asthma), renal failure, and human immunodeficiency virus (HIV).

Pharmacy claim variables. Using claims in the first 6 months of the study, pharmacy claim variables included: antipsychotics, antidepressants, mood stabilizers, sedative/hypnotics, and anxiolytics. If the patient had at least one prescription filled for a medication from one of these categories, he or she was considered positive for that dichotomous variable.

Dependent variable. The dependent variable was the total number of ED visits during the second 6 months of the study period.

\section{Statistical analysis}

Statistical procedures included difference of means tests, Pearson correlation, and ordinary least squares multiple regression. To assess the statistical significance of the difference in ED utilization in the second 6 months where there was a dichotomous independent variable (e.g., presence of alcohol abuse), $t$ tests were performed. To gauge the statistical significance of the difference in ED utilization in the second 6 months where there was a continuous independent variable (e.g., number of hospitalizations), Pearson correlation coefficients were calculated. Using significantly related variables, multiple regression analysis was used to find the unique effect of each independent variable as a predictor of ED utilization. Variables with significant $F$ ratios in the regression analysis were retained as factors in the risk model, and their additive and cumulative effects were evaluated. All analyses were performed using SAS (Statistical Analysis Software) release 8.1.

\section{RESULTS}

\section{Demographics}

A total of 764 patients had a documented medical claim for a psychotic disorder (ICD-9CM 295.xx-299.xx). The mean age in this population was 44.5 years (SD 14.43), with a range from 18 to 92 years, and more than half were 
female (58\%). The ethnicity breakdown was $1 \%$ Asian, 54\% African American, 11\% Hispanic, $30 \%$ Caucasian, $2 \%$ other, and $2 \%$ unknown. Approximately $46 \%$ of patients had a diagnosis for schizophrenia (295.xx), 32\% for affective psychoses (296.xx), 3\% for paranoid states (297.xx), 36\% for other psychoses (298.xx), and $1 \%$ for psychosis with origination specific to childhood (299.xx). The sum of the percentages for diagnosis identification is greater than $100 \%$ (764 patients) as a result of patients with multiple diagnoses among the relevant ICD-9-CM codes.

Each medical claim contained up to four diagnosis codes. For $54 \%$ of the patients, their identifying psychosis code was found in the primary diagnosis field, while the remaining patients had their identifying diagnosis in one of the other three fields. Of the five potential types of psychotic diagnoses measured in this study, 647 (85\%) patients had claims for only one of these diagnoses, while
$117(15 \%)$ patients had claims captured in more than one of the five possible identifying diagnoses.

The primary diagnoses for ED visits during the last 6 months of the study period were examined to investigate the main reasons patients sought treatment through the ED. Although a variety of causes were found when assessing ED visits, a few broad diagnosis categories were identified with the highest frequencies. From a total of 1,259 ED visits during the last 6 months of the study period, approximately $34 \%$ were due to ill-defined symptoms, signs, and conditions. Close to $17 \%$ of ED visits were attributable to injuries and poisonings, while mental disorders accounted for the primary diagnosis in $13 \%$ of the ED cases. Diseases related to the musculoskeletal system and connective tissue were the cause for $7 \%$ of cases, while diseases of both the digestive and respiratory systems were found as the primary diagnosis in approximately $5 \%$ of ED visits.

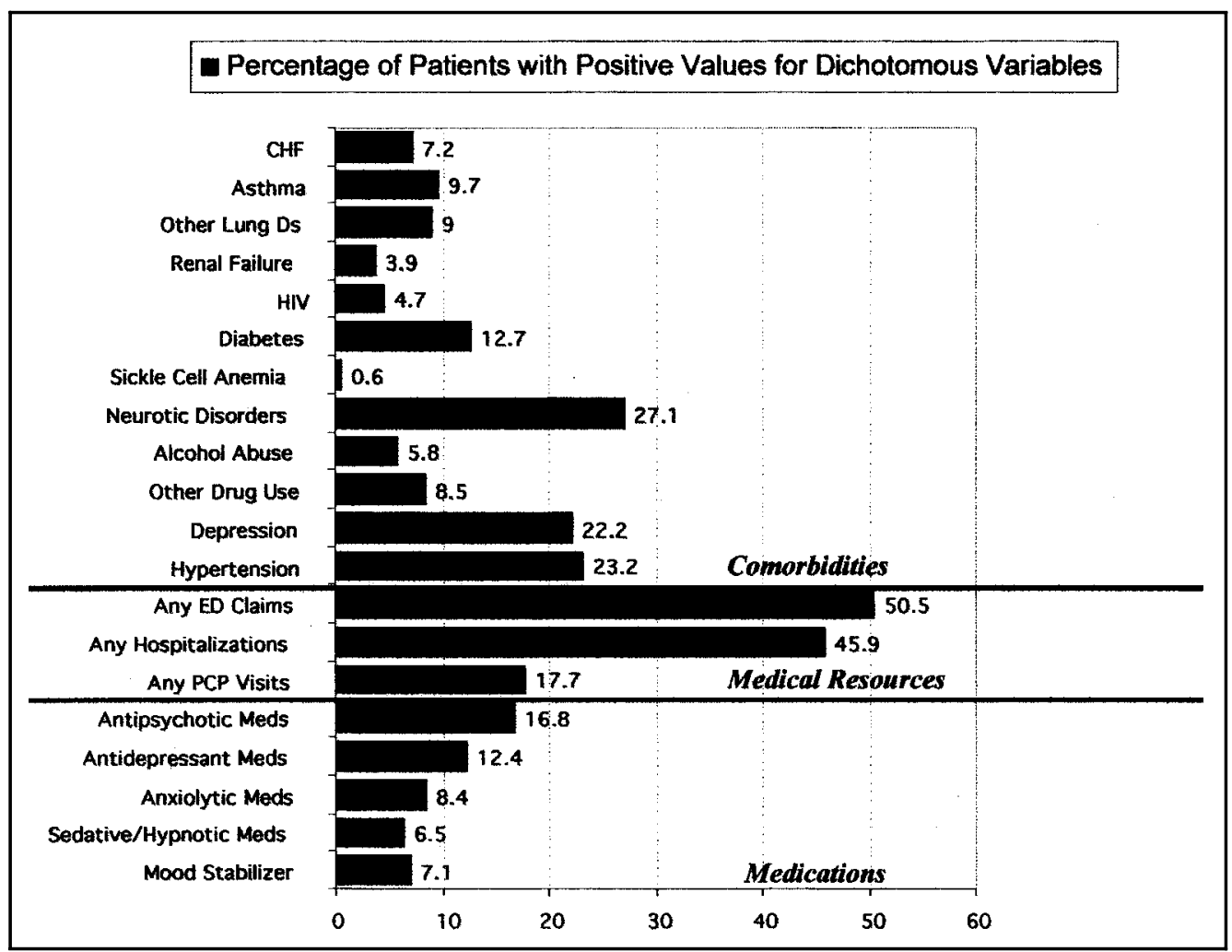

FIG. 1. For each dichotomous variable used in the study, the percentage of patients based on the entire study population $(n=764)$ having a medical or pharmacy claim for that variable is represented by the associated bar graph. Percentages for comorbidity variables were derived from the entire study year, while percentages for medical resources and medications were derived from the first 6 months only. 


\section{Independent variables}

All independent dichotomous variables tested in the model and their frequencies are depicted in Figure 1. The dichotomous variable frequencies are based on the number of patients positive for the variable. The five variables found most often were at least one ED claim during the first 6 months, at least one hospitalization, neurotic disorders, HTN, and depression. For continuous variables, the mean for the total patient population was evaluated as well as the mean using only patients with non-zero values per variable. These results are shown in Figure 2 and demonstrate a large difference between the mean for all patients and the mean for non-zero cases. For example, the average number of hospital days per patient in the total study population was 5.62 days, but after eliminating those patients who had zero hospital days reported, the average was 12.58 days.

\section{Regression and risk model results}

The following variables were found to have a significant association $(p<0.05)$ with ED vis- its based on a $t$ test or Pearson correlation test: younger patients, African American ethnicity, number of hospitalizations, at least one hospitalization, number of hospital days, ED visits in the first 6 months, patients not prescribed antipsychotic medication, alcohol abuse, anemia, congestive heart failure (CHF), depression, diabetes, HIV, HTN, neurotic disorders, other lung disease (besides asthma), other drug abuse (besides alcohol), and renal failure. The three variables related to hospitalizations were significantly related with one another; therefore only the variable having the highest correlation to ED visits, number of hospitalizations, was used in the regression analysis. From the ordinary least squares multiple regression analysis, the four variables found to be significantly $(p<0.05)$ associated with ED visits were presence of alcohol abuse, presence of depression, number of ED visits during the first 6 months, and number of hospitalizations during the first 6 months (Table 1).

The parameter estimates express the relationship of each variable to an increase in ED visits during the second 6 months. For exam-

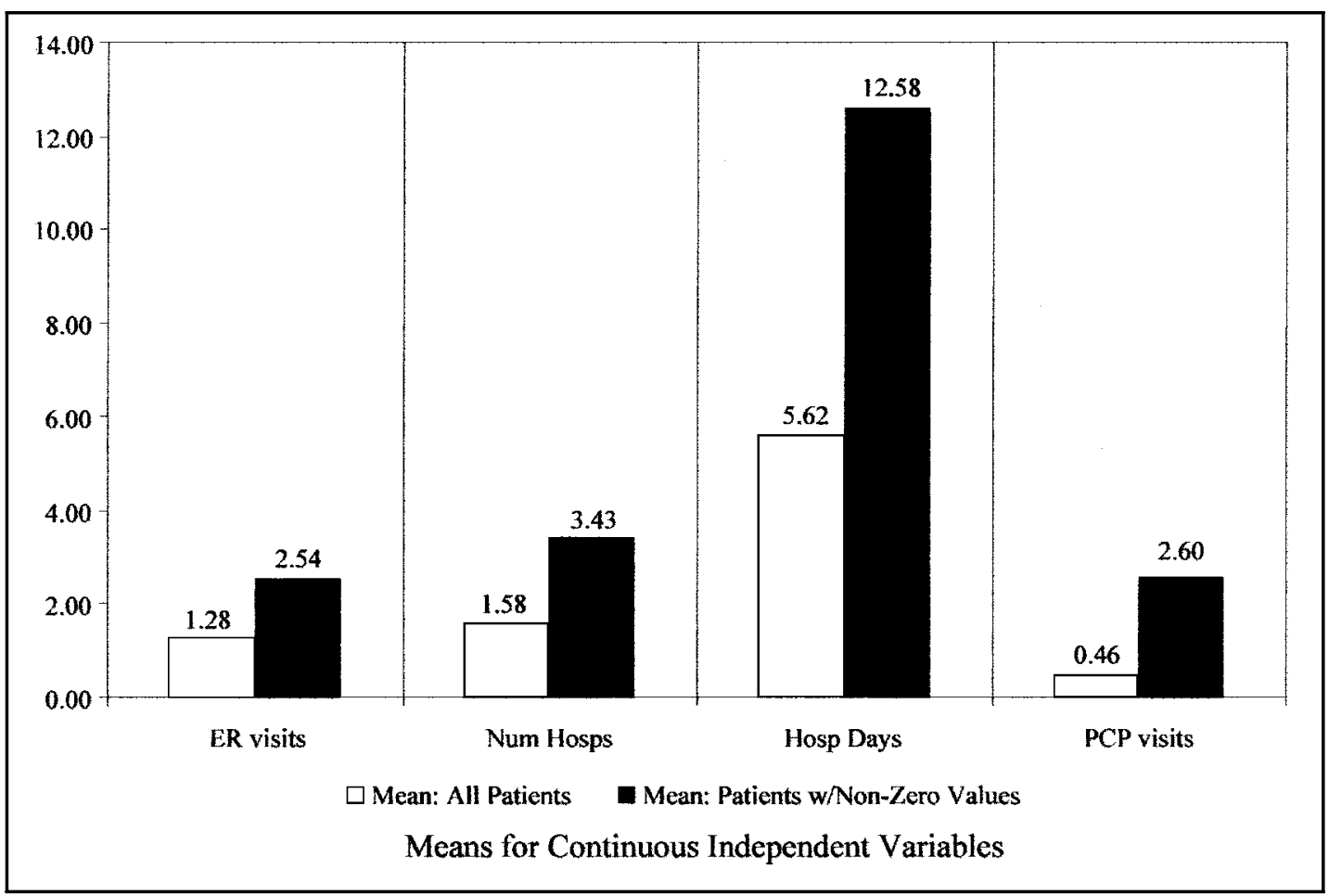

FIG. 2. The mean count for each continuous independent variable used in this study is represented by a bar graph. Each variable has two groups of mean scores for either all patients in the study or only those patients with a nonzero value for that variable (excluding patients with zero cases for each variable). ER, emergency room. 


\begin{tabular}{|c|c|c|c|c|c|c|c|}
\hline \multirow[b]{3}{*}{ Variable description } & \multirow{2}{*}{\multicolumn{2}{|c|}{$\mathrm{t}$ test }} & \multirow{2}{*}{\multicolumn{2}{|c|}{$\begin{array}{l}\text { Pearson } \\
\text { correlation }\end{array}$}} & \multicolumn{3}{|c|}{ Regression analysis } \\
\hline & & & & & & & Parameter \\
\hline & $\mathrm{t}$ value & $\mathrm{p}$ value & $\mathrm{r}$ value & $\mathrm{p}$ value & $\mathrm{t}$ value & $\mathrm{p}$ value & estimate \\
\hline Presence of alcohol abuse & 3.11 & 0.0033 & - & - & 2.59 & 0.0097 & 0.288 \\
\hline Presence of depression & 4.50 & $<0.0001$ & - & - & 3.54 & 0.0004 & 0.565 \\
\hline $\begin{array}{l}\text { Number of ED visits during } \\
\text { 1st } 6 \text { months }\end{array}$ & - & - & 0.6104 & $<0.0001$ & 13.79 & $<0.0001$ & 0.452 \\
\hline $\begin{array}{l}\text { Number of hospitalizations } \\
\text { during 1st } 6 \text { months }\end{array}$ & - & - & 0.5160 & $<0.0001$ & 9.56 & $<0.0001$ & 0.021 \\
\hline
\end{tabular}

ple, a patient with a diagnosis of alcohol abuse would have $28.8 \%$ more ED visits versus patients without that diagnosis. For continuous variables, an increase of the independent variable predicts additional ED utilization. For example, an additional hospitalization during the first 6 months would increase the risk by $2 \%$ for an ED visit during the following 6 months.

\section{Combined risk factor effects}

The results of the regression model described above were used to identify and evaluate the additive and cumulative effects of the four risk factors (Table 2). A pattern is seen with these effects: As the number of risk factors increases, there is a consistent rise in the number of ED visits during the second 6 months. Where none of the selected risk factors is present, the mean number of ED visits during the second half is 0.58; where all four risk factors are present, the mean number of ED visits during the second half is 8.50 . Only seven of the 764 cases do not follow the linear pattern of increasing risk factors with increasing ED visits (five cases with

\begin{tabular}{|c|c|c|c|c|c|c|c|}
\hline$A l c A b$ & Deprn & $E D V 1 H^{1}$ & Hos $1 H^{1}$ & NumRF & $E D V 2 H$ & $\begin{array}{c}\text { Cases } \\
(\mathrm{n}=764)\end{array}$ & $\begin{array}{c}\% \text { of cases } \\
(100 \%)\end{array}$ \\
\hline Yes & Yes & $2-29$ & $3+$ & 4 & 8.50 & 6 & $0.8 \%$ \\
\hline Yes & No & $2-29$ & $3+$ & 3 & 6.00 & 9 & $1.2 \%$ \\
\hline No & Yes & $2-29$ & $3+$ & 3 & 5.12 & 26 & $3.4 \%$ \\
\hline Yes & Yes & $0-1$ & $3+$ & 3 & 4.33 & 3 & $0.4 \%$ \\
\hline Yes & Yes & $2-29$ & $0-2$ & 3 & 3.71 & 7 & $0.9 \%$ \\
\hline No & No & $2-29$ & $3+$ & 2 & 2.88 & 33 & $4.3 \%$ \\
\hline No & Yes & $0-1$ & $3+$ & 2 & 2.45 & 11 & $1.4 \%$ \\
\hline Yes & No & $0-1$ & $3+$ & 2 & 1.80 & 5 & $0.7 \%$ \\
\hline No & Yes & $2-29$ & $0-2$ & 2 & 1.69 & 39 & $5.1 \%$ \\
\hline No & No & $2-29$ & $0-2$ & 1 & 1.62 & 91 & $11.9 \%$ \\
\hline No & No & $0-1$ & $3+$ & 1 & 1.28 & 46 & $6.0 \%$ \\
\hline Yes & No & $2-29$ & $0-2$ & 2 & 1.20 & 5 & $0.7 \%$ \\
\hline No & Yes & $0-1$ & $0-2$ & 1 & 1.20 & 76 & $9.9 \%$ \\
\hline Yes & No & $0-1$ & $0-2$ & 1 & 0.86 & 7 & $0.9 \%$ \\
\hline No & No & $0-1$ & $0-2$ & 0 & 0.58 & 398 & $52.1 \%$ \\
\hline Yes & Yes & $0-1$ & $0-2$ & 2 & 0.50 & 2 & $0.3 \%$ \\
\hline \multicolumn{8}{|c|}{$\begin{array}{l}\text { This model demonstrates how ED use in the study population increased as the number of risk factors increased } \\
\text { per patient. Cases and percentages represent the amount of the population having the specified number of risk } \\
\text { factors. AlcAb, alcohol abuse; Deprn, depression; EDV1H, ED visits during first half; Hos1H, hospitalizations dur- } \\
\text { ing first half; NumRF, number of risk factors; EDV2H, ED visits during second half. } \\
{ }^{1} \text { To produce a parsimonious model, the continuous variables "ED visits" and "Hospitalizations during 1st half" } \\
\text { were broken into two categories at points of inflection in the curves of their effects on ED visits during the second } \\
\text { half. }\end{array}$} \\
\hline
\end{tabular}


alcohol abuse and more than two ED visits and two cases with alcohol abuse and depression). Patients with all four of these risk factors had the highest likelihood of using ED services. As the number of risk factors decrease, patients had a decreasing chance of ED use.

\section{DISCUSSION}

High use of the ED has been documented and studied previously in the general psychotic population. $8,9,14$ One commonly recognized predictor for high use of either acute inpatient services or the ED was having a history of past hospitalizations. ${ }^{914}$ Another previous finding consistent with the results of this study is that patients having a severe diagnosis, such as alcoholism or schizophrenia, are frequent repeat users of ED services. ${ }^{8}$

When presenting at the ED, only $54 \%$ of the patients in this study had a psychosis diagnosis as their primary problem. This supports the concept that these patients have multifaceted health issues and may benefit greatly from the specialized, targeted care of case management. The behavioral health carve-out model may be another reason that only half the patients in our study came to the ED with psychosis being the primary cause. The mental health care they receive is provided by the behavioral health plan and is not captured in the records used for this study. Therefore, practitioners in the ED may not be aware that these patients have additional medical problems related to psychosis that may be directly or indirectly affecting their ED use.

Additionally, owing to the carve-out model, this study only included patients with a psychotic diagnosis by a medical (not behavioral) service provider. During protocol development, the possibility of using pharmacy claims to identify patients with psychosis was discussed in order to expand the patient population and partially adjust for the carve-out model limitation. This idea was not implemented owing to the multiple potential uses of antipsychotic medication and therefore the risk of inaccurately identifying patients as psychotic. This limitation may have also excluded patients with psychosis who were prescribed antipsychotics but did not have an identifiable diagnosis code as a medical claim. Despite these limitations, over 700 members with a diagnosis of psychosis were identified. The predictive model that was developed should prove to be of use in effectively targeting disease management efforts aimed at reducing inappropriate ED use and increasing primary care access for these members. The model should be validated in other populations and may prove useful for MMCOs that do not operate under behavioral health carve-outs, as well as other non-MMCOs seeking to identify candidates for disease management efforts focused on use of ED services.

By targeting patients with a higher number of risk factors there is the potential for early intervention with the goal to improve quality of care. The benefits may be twofold: The patient with multiple problems will be identified and treated appropriately, while preserving emergency health care resources for urgent cases.

\section{ACKNOWLEDGMENTS}

The authors wish to thank David B. Nash, M.D., M.B.A., from the Office of Health Policy and Clinical Outcomes at Thomas Jefferson University for his support and review of this project. Dr. Girts conducted this research as a Janssen Outcomes Research Fellow at Thomas Jefferson University, and the project was funded by Janssen Pharmaceutica, Inc.

\section{REFERENCES}

1. DeParle NA, adm. A profile of Medicaid chartbook 2000. Washington, DC: U.S. Department of Health and Human Services, Health Care Financing Administration, September 2000. Available at: http:/ / www. hcfa.gov/stats/2Tchartbk.pdf. Accessed January 2002.

2. Smith V, Ellis E. Medicaid budgets under stress: survey findings for state fiscal year 2000, 2001, 2002. Report no. 4020. Washington, DC: Kaiser Commission on Medicaid and the Uninsured, October 2001. Available at: http://www.kff.org/content/2001/10252001/ 4020.pdf. Accessed February 2002.

3. Medicaid and managed care. Report no. 206803.Washington, DC: Kaiser Commission on Medicaid and the Uninsured, December 2001. Available at: http: / / www.kff.org/content/2001/206803/206803.p df. Accessed February 2002. 
4. Gold M. Making managed care research relevant. Health Serv Res 1999;33:1639-1650.

5. Butler PA. Medicaid HMO enrollees in the emergency room: use of nonemergency care. Med Care Res Rev 1998;55:78-98.

6. Cunningham PJ, Clancy CM, Cohen JW, Wilets M. The use of hospital emergency departments for nonurgent health problems: a national perspective. Med Care Res Rev 1995;52:453-474.

7. Taube CA, Goldman HH, Salkever D. Medicaid coverage for mental illness: balancing access and costs. Health Affairs 1990;9:5-18.

8. Ellson JM, Blum NR, Barky AJ. Frequent repeaters in a psychiatric emergency service. Hosp Community Psychiatry 1989;40:958-960.

9. Havassy BE, Hopkin JT. Factors predicting utilization of acute psychiatric inpatient services by frequently hospitalized patients. Hosp Community Psychiatry 1989;40:820-823.

10. Sullivan PF, Bulik PF, Forman SD, Mezzich JE. Characteristics of repeat users of a psychiatric emergency service. Hosp Community Psychiatry 1993;44: 376-380.

11. Carver T, Homa-Lowry J, Zaseck R. Reduce costs, improve outcomes with community case management. Hosp Case Manage 2001;9:33-36.
12. Carver T. Goals for a community-based case management program [chart]. Hosp Case Manage 2001;9:35. [In: Carver T, Homa-Lowry J, Zaseck R. Reduce costs, improve outcomes with community case management. Hosp Case Manage 2001;9:33-36.]

13. Practice Management Information Corporation. 4th ICD-9-CM: International Classification of Diseases, 9th revision, clinical modification. Los Angeles: Practice Management Information Corporation, 1992: 125-129.

14. Surles RC, McGurrin MC. Increased use of psychiatric emergency services by young chronic mentally ill patients. Hosp Community Psychiatry 1987;38:401-405.

Address reprint requests to:

Tammy K. Girts, Pharm.D. Health Economics

Boehringer Ingelheim Pharmaceuticals, Inc. 900 Ridgebury Road Ridgefield, CT 06877

E-mail: tgirts@rdg.boehringer-ingelheim.com 